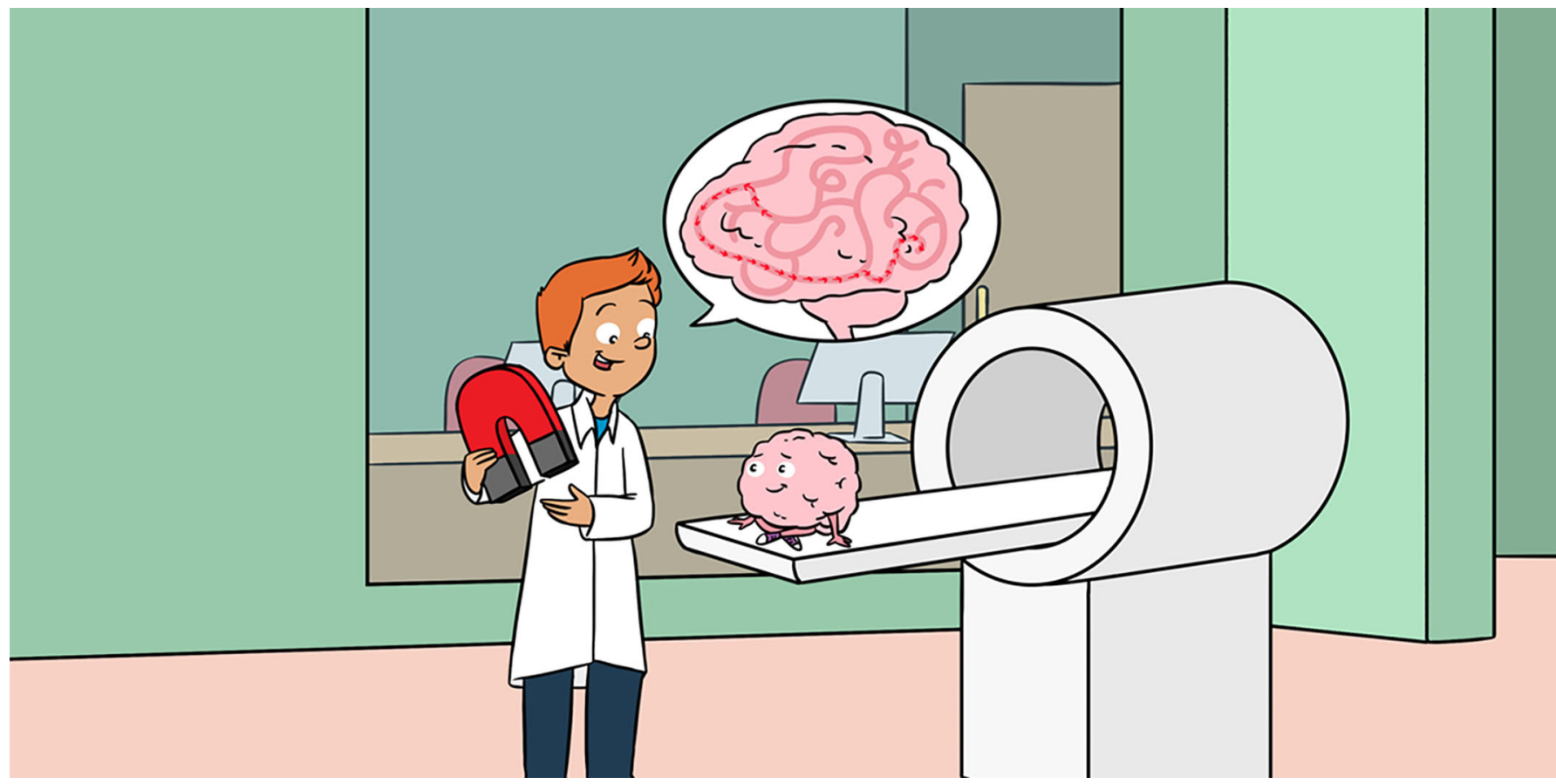

\title{
A WINDOW INTO YOUR BRAIN: HOW FMRI HELPS US UNDERSTAND WHAT IS GOING ON INSIDE OUR HEADS
}

\section{Udochukwu Amanamba *, Andrew Sojka ", Savion Harris ", Marisa Bucknam and Jay Hegdé}

Department of Neuroscience and Regenerative Medicine, Medical College of Georgia, Augusta University, Augusta, GA, United States

\section{YOUNG REVIEWERS:}

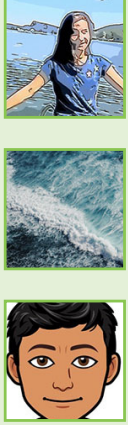

ISABELLA

AGE: 13

ALINE

AGE: 13

LUCAS

AGE: 15
The brain-the last frontier of modern science. Despite many technological advances, we still know little about how the brain works. Luckily, the development of a technique called functional magnetic resonance imaging (fMRI) is slowly helping change this. fMRI can measure brain activity without opening the skull or exposing the brain cells to harmful radiation. By using the blood's magnetic properties, fMRI can detect changes in blood flow related to brain activity, allowing scientists and physicians to tell which regions of the brain are more active than others. Currently, researchers use fMRI to study various aspects of brain activity in health and disease. Scientists continue to push the boundaries of the fMRI technique and combine it with other techniques to obtain an even better understanding of brain function and dysfunction. 
Figure 1

Components of an MR scanner [2]. MRI and fMRI scans take place within the same MRI scanner.

\section{MAGNETIC FIELD}

Field that occurs around a magnet, which exerts a force that attracts or repels other magnetic objects

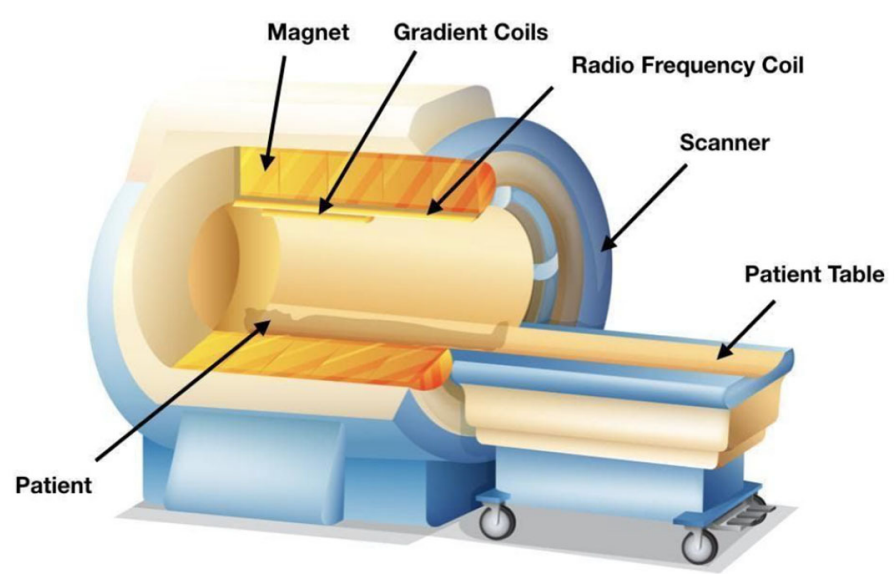

Figure 1

\section{WHAT IS FMRI AND HOW DOES IT WORK?}

"Do you ever look at someone and wonder, 'what is going on inside their head?"' This famous question posed by the movie character Joy, from the Disney Pixar movie Inside Out, is a puzzle doctors and scientists seek to solve daily with the help of brain imaging. Brain imaging allows doctors and scientists to view the interior structures of the brain without ever opening the skull. There are several brain-imaging techniques. One of them is called magnetic resonance imaging (MRI), which looks at the structure of the brain and another is functional magnetic resonance imaging (fMRI), which looks at the brain's function.

fMRI measures brain activity by tracking changes in blood flow to the brain. $\mathrm{fMRI}$ is derived from structural MRI. In fact, they both use the same machine, called a magnetic resonance scanner (Figure 1). MRI technology is used to create detailed, 3D images of the internal structure of an object using magnetic fields and radio waves [1]. MRI can be used to study body parts other than the brain, and even non-living objects. For example, MRI could be used by an archaeologist to take pictures of the inside of a fossil. fMRI can also be used to image body parts other than the brain. In medicine, brain MRI and fMRI are used to help recognize illnesses, plan treatments, and study the underlying causes of diseases and disorders.

MR scanners work by taking pictures of the brain one thin layer at a time. The pictures are then stacked like pancakes to create a full picture of the region being imaged. How is this possible, you may ask? The human body is made up of billions of molecules, including water molecules, which can be detected by the MRI machine. Atoms in all molecules, including water molecules $\left(\mathrm{H}_{2} \mathrm{O}\right)$, contain protons [1]. Protons are like tiny magnets [1]. In the absence of a very strong magnetic field (that is, when we are outside the MR scanner), protons in our body are oriented in random directions (Figure 2A). When we lie 
Figure 2

Molecules in the body during an MRI scan. (A) Molecules in the body, which act like tiny magnets, are oriented in random directions in the absence of a strong magnetic field. (B) MR scanners produce a strong magnetic field (dashed red arrow) that forces the molecules in the body to align with the field. (C) RF coils transmit radio frequencies (dotted aqua arrow)

throughout areas of the body being imaged.

The molecules become realigned in between the directions of the magnetic fields of the RF coil and the scanner. (D) When radio frequencies are no longer transmitted, the molecules revert, or "relax," to their original alignment with the scanner's magnetic field, releasing energy in the form of electromagnetic waves (inset at top right). The energy that is released can be analyzed to create an image of the body part.

\section{RADIO FREQUENCY} WAVES

\section{Special}

electromagnetic signals created by radio

frequency coils to realign protons from the magnetic field of the MRI

scanner's magnet.

ELECTROMAGNETIC SIGNALS

Signals created through the vibrations of magnetic fields and electric fields.
A

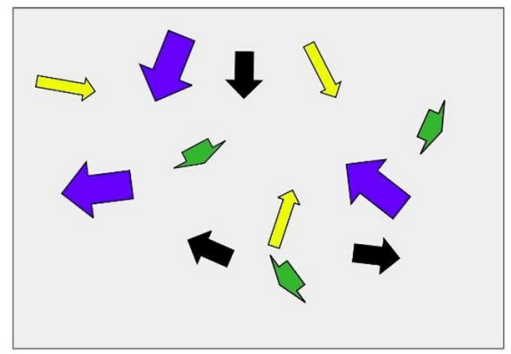

C

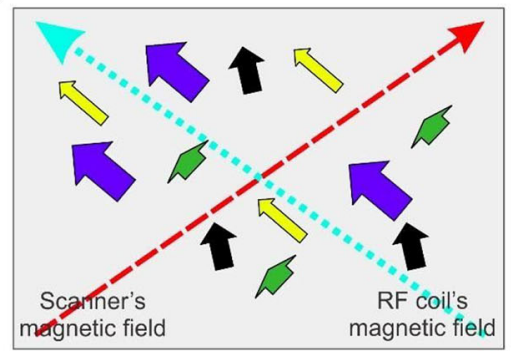

B

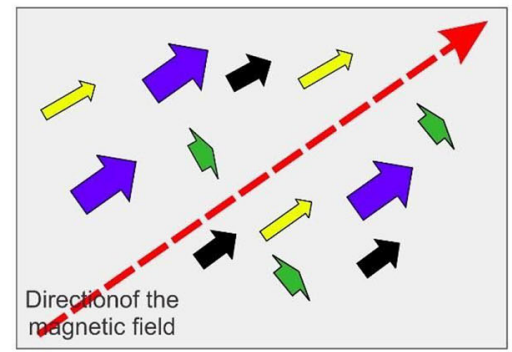

D

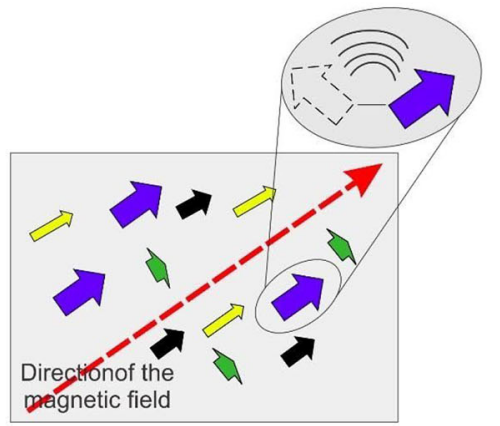

Figure 2

inside the scanner, its strong magnetic field, which is usually tens of thousands of times the strength of the earth's magnetic field, forces these protons to align with the field, although we cannot feel this at all (Figure 2B). The gradient coil (see Figure 1) helps the scanner operators determine exactly where our body is inside the scanner.

Then, the radio frequency (RF) coils transmit radio frequency waves throughout the areas of the body that are being imaged, to realign these protons yet again, but temporarily (Figure $2 \mathrm{C}$ ). RF coils may be part of the MRI machine for full-body scan or worn as a special headband when just imaging the brain. When the radio frequencies are no longer transmitted, the protons "relax" into their original alignment with the scanner's magnetic field. In doing so, the protons release the energy that was tugging them in the RF coil's direction (like releasing a slingshot), in the form of electromagnetic signals (Figure 2D).

In the same way that millions of water droplets can create a puddle, the signals from millions of protons, when carefully anaylzed, can come together to create a detailed image of the body [1]. While MRI only takes pictures of the brain structure, fMRI shows the activity (or function) of the brain, by comparing the blood flow under different conditions.

\section{NEURONS: THE BUILDING BLOCKS OF OUR BRAINS}

The brain helps us understand and respond to the world around us. It enables us to interpret things we see, touch, hear, and taste, and regulates our body's responses to the external environment. It does 
Figure 3

Movement of oxygenated and deoxygenated hemoglobin during neural activity. (A) When neural activity increases in region of the brain, more energy is used by that brain region, and hemoglobin becomes deoxygenated as it gives oxygen to the cells that need it. (B) To replenish this energy, more oxygenated blood is transported to that brain region.

\section{NEURONS}

Nerve cells that send and receive electrical and chemical signals across long distances, especially in the brain

\section{HEMOGLOBIN}

Iron-containing protein inside red blood cells that captures oxygen and transports it to the tissues of the body.

\section{Deoxygenated hemoglobin * Oxygenated hemoglobin}

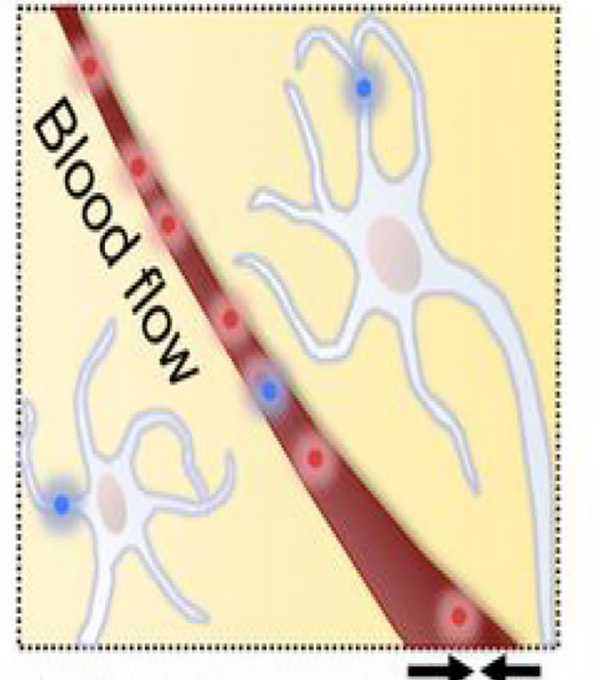

A Less neural activity

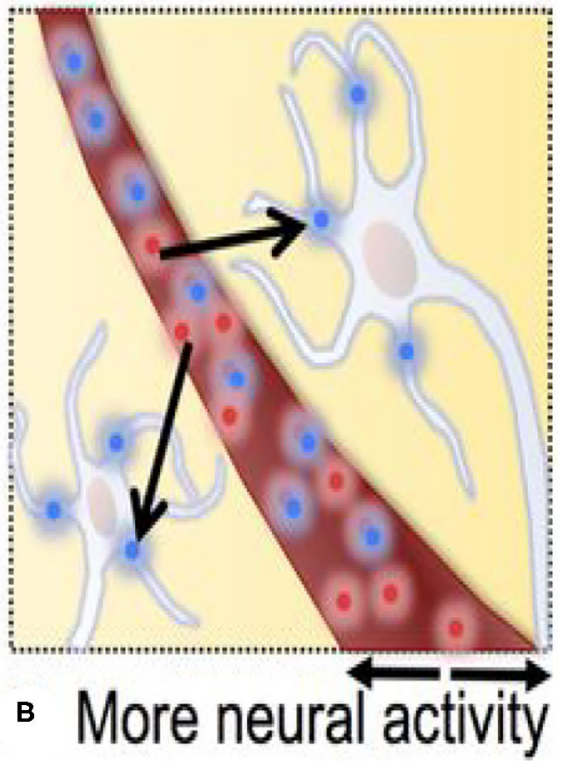

Figure 3 all this through networks of tiny cells called neurons, which process and transmit information between the brain and the rest of the body [3]. When the brain is faced with a task, such as remembering an idea, neurons responsible for that activity become more active than other neurons around them. They do this by creating chemical and electrical signals and transferring them from one neuron to another. This process is referred to as neural activity or brain activity.

\section{HOW DOES FMRI MEASURE BRAIN ACTIVITY?}

Neural activity requires energy. Like other cells in the body, neurons generate energy by using oxygen to break down sugar. When neural activity increases in a part of the brain, more energy is used. To replenish this energy, more oxygen-carrying blood is transported to that brain region. The blood transports oxygen using a molecule called hemoglobin. Hemoglobin contains iron, which gives it magnetic properties, like a tiny iron filing. Depending on whether hemoglobin is carrying oxygen or not (that is, whether it is oxygenated or deoxygenated), it has slightly different magnetic properties. Thus, greater neural activity results in greater flow of oxygenated blood (Figure 3), so that brain regions that are more active are slightly more magnetic. This results in slightly different patterns of electromagnetic waves.

fMRI detects brain activity by measuring the changes in both the amount of oxygen in the blood and the amount of blood flow $[4,5]$. This measurement is known as blood-oxygen-level-dependent 
Figure 4

Brain activity in dogs when they are expecting a reward [6]. (A) A side view of a dog's head. The blue plane shows the plane in which the brain images were obtained.

(B) The BOLD activity in two healthy female dogs named Callie and McKenzie was measured while they watched their handler's hand signals for reward vs. no-reward. fMRI images are on the left, and the structural MRI images are on the right. The images are a top-down view of the brain taken at the plane shown in (A). The caudate region (CD) lights up in the fMRI images, meaning that it is activated, and the green arrow shows the location of the CD in the corresponding structural MRI images. $\mathrm{R}$ and $\mathrm{L}$ denote the right and left sides of the dog. The color bar indicates the level of activity, with red being highest and blue being lowest.
A

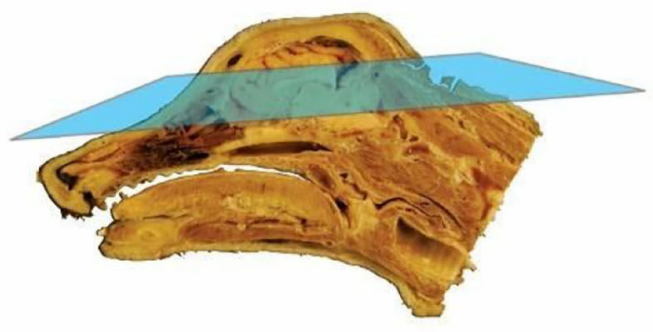

B

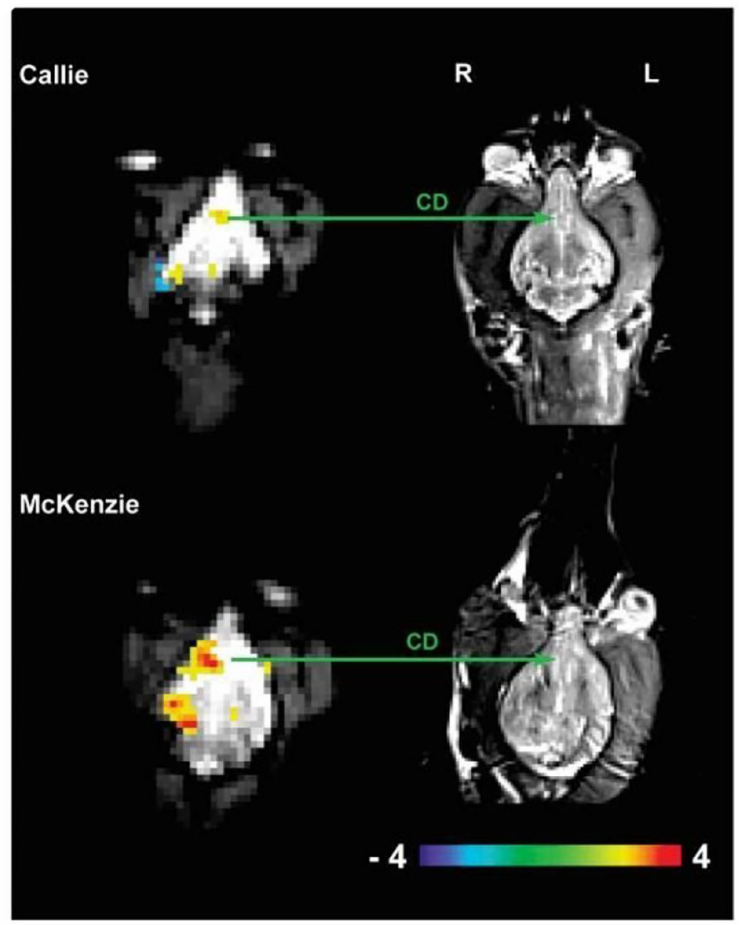

Figure 4

activity (BOLD activity). In other words, BOLD activity is a convenient stand-in for brain activity: fMRI measures brain activity indirectly by measuring BOLD activity. This is somewhat akin to figuring out where and when lightning happened by listening to the thunder.

\section{"BOLD" INSIGHTS INTO BRAIN FUNCTION}

Over the last three decades or so, researchers have using BOLD MR imaging to begin to answer Joy's question about what is going on inside people's heads. The power of fMRI to peer into the otherwise impenetrable depths of brain function, in humans or animals, is illustrated beautifully by the work of Dr. Gregory Berns and his colleagues. They compared the BOLD activity in the brains of dogs in response to two different hand signals by their handlers: one that told the dogs they were going to get a tasty food reward, and another that told them they would not get a reward (Figure 4A). 
CAUDATE NUCLEUS

A region of the brain which, among other things, responds to rewards.
They found that a region deep in the brain, the caudate nucleus (CD), was more active when the dogs saw the signal for the reward than when they saw the no-reward signal (Figure 4B). Previous studies in humans and other species have shown that the same brain region is activated in humans when we are expecting a reward. So, there is an answer to Joy's question in this example: what goes on in a dog's head is remarkably similar to what goes on in our own heads in similar situations, and can be imaged using fMRI in both cases [6]. This remarkable insight would not have been possible without being able to "read" the dogs' brains using fMRI!

\section{IMPACT ON OTHER FIELDS, INCLUDING MEDICINE}

The development of $\mathrm{MRI}$ and $\mathrm{fMRI}$ has allowed us to make major strides in understanding brain function. Part of this is because MRI and $\mathrm{fMRI}$, unlike X-rays for instance, do not damage the brain cells with harmful radiation, and can determine the location of brain activity with more precision. Furthermore, MRI can be used to study a wide variety of things in addition to humans, including animals, plants, other organisms, and even fossils that do not have blood.

However, the biggest impact of MRI and fMRI, outside of pure research, has been in understanding brain diseases. For instance, just a few years ago, we were only able to track Alzheimer's disease in the later stages, when most of the damage to the brain is irreversible. With the development of fMRI, early detection of Alzheimer's is becoming increasingly possible. Individuals with Alzheimer's tend to have less activity in certain parts of their brains compared to healthy people. Hence, doctors can use fMRI to diagnose Alzhiemer's early, by detecting abnormal or lower levels of activity in these regions of the brain before the disease worsens.

Also, fMRI scans are now able to distinguish between a brain with or without autism, with $97 \%$ accuracy [7]. fMRI studies have shown patterns of decreased activity in the part of the brain important for planning, problem solving, and interpreting social interactions. Moreover, scans of patients with autism have shown fewer connections between the two halves of the brain. Thus, by systematically comparing brain function in patients with certain brain-related diseases to the brain function of healthy patients, rapid progress is being made in understanding these diseases.

\section{WHAT IS NEXT?}

Despite all the progress, a number of challenges remain. For instance, we still know very little about how the brain develops in early childhood. Currently, fMRI machines are dark, noisy, and scary, making them unsuitable for infants and people scared of cramped 
spaces. They also take too long to create images and their "cameras" are extremely sensitive to movement, making the images blurry. Furthermore, fMRI is expensive, not portable, and requires a lot of training for doctors and scientists to use. Researchers are working to solve these problems. Another major challenge is to improve the speed of the MR imaging process and to improve the quality of the images. These and several other remaining challenges can seem forbidding, but they are really future opportunities for today's young, aspiring minds.

\section{REFERENCES}

1. Khan Academy. Magnetic Resonance Imaging (MRI). Available online at: https://www.khanacademy.org/test-prep/mcat/physical-processes/proton -nuclear-magnetic-resonance/a/magnetic-resonance-imaging-mri (accessed October 7, 2020).

2. Vecteezy. 2019. Vector Graphics for Everyone. Available online at: www.vecteezy.com/ (accessed October 7, 2020).

3. BrainFacts/sfn. 2012. The Neuron. Available online at: http://www.brainfacts.org /brain-anatomy-and-function/anatomy/2012/the-neuron (accessed October 7 , 2020).

4. Kids Encyclopedia Facts. Functional Magnetic Resonance Imaging Facts for Kids. Available online at: https://kids.kiddle.co/Functional_magnetic_resonance_ imaging (accessed October 7, 2020).

5. Neuroscience of Attention \& Perception Laboratory (NAPL), Princeton University. NAPL Kids Project. Available online at: https://scholar.princeton.edu/kidsrock (accessed October 7, 2020).

6. Berns, G. S., Brooks, A. M., and Spivak, M. 2012. Functional MRI in awake unrestrained dogs. PLOS ONE. 7:e38027. doi: 10.1371/journal.pone.0038027

7. Oaklander, M. 2014. Autism: fMRI Brain Scans Can Predict Autism With $97 \%$ Accuracy. Available online at: https://time.com/3614487/fmri-autism-diagnosis/ (accessed October 7, 2020).

SUBMITTED: 12 July 2019; ACCEPTED: 11 September 2020; PUBLISHED ONLINE: 04 November 2020.

EDITED BY: Sabine Kastner, Princeton University, United States

CITATION: Amanamba U, Sojka A, Harris S, Bucknam M and Hegdé J (2020) A Window Into Your Brain: How fMRI Helps Us Understand What Is Going on Inside Our Heads. Front. Young Minds 8:484603. doi: 10.3389/frym.2020.484603

CONFLICT OF INTEREST: The authors declare that the research was conducted in the absence of any commercial or financial relationships that could be construed as a potential conflict of interest.

COPYRIGHT @ 2020 Amanamba, Sojka, Harris, Bucknam and Hegdé. This is an open-access article distributed under the terms of the Creative Commons Attribution License (CC BY). The use, distribution or reproduction in other forums 

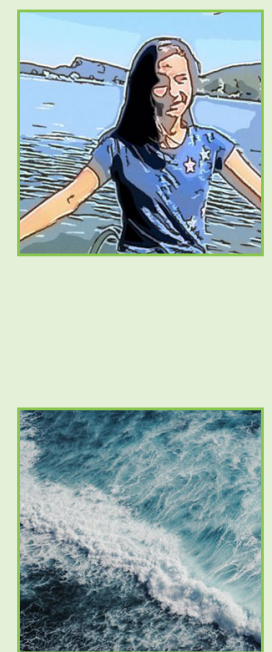

is permitted, provided the original author(s) and the copyright owner(s) are credited and that the original publication in this journal is cited, in accordance with accepted academic practice. No use, distribution or reproduction is permitted which does not comply with these terms.

\section{YOUNG REVIEWERS}

\section{ISABELLA, AGE: 13}

My name is Isabella and I am 13 years old. I was born in New York and I live in Switzerland. I love art, reading books, singing, dancing, and playing the piano. My favorite sport is swimming. I also have great interest in math, nature, and science, especially everything about space, time, and matter. In the future, I would like to be an aeronautic engineer or an architect. I am always very curious to learn more about the world around us.

\section{ALINE, AGE: 13}

My name is Aline, I am 13 years old. My favorite hobbies include theater, playing the clarinet, drawing, and reading. I am fascinated with Greek mythology; my favorite books include the Harry Potter and the Percy Jackson series. At school, I really enjoy Maths and Science.

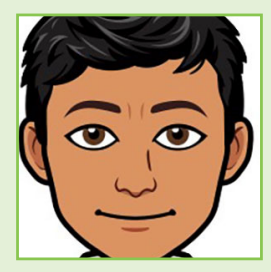

\section{LUCAS, AGE: 15}

My name is Lucas, I am a tenth grader at the Brookline High School. I speak English, Spanish, and I am currently taking Chinese and I have been learning it since second grade. I like playing and watching soccer and also playing Xbox.

\section{AUTHORS}

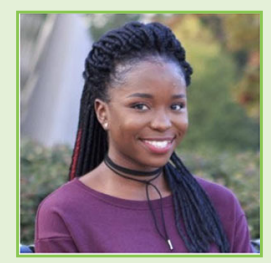

\section{UDOCHUKWU AMANAMBA}

I am an undergraduate student at Augusta University, Augusta, Georgia (U.S.A.) studying Psychology and Biology with a pre-medicine concentration. I enjoy learning about the brain and neurological disorders, and hope to soon provide medical care to people suffering from neurological disorders. I am currently a research intern in Dr. Jay Hegde's lab and a participant in the Army Educational Outreach Program (AEOP): Undergraduate Research Apprenticeship Program (URAP). In my spare time, I enjoy singing, traveling, reveling in nature, spending time with my family, and pranking my friends. *uamanamba@augusta.edu

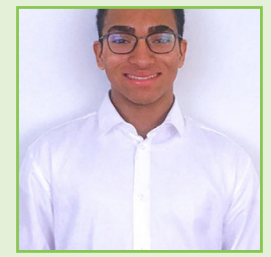

\section{ANDREW SOJKA}

I am a rising junior attending Poolesville High School in Poolesville, Maryland (U.S.A.), interested in studying neuroscience, medicine, and journalism. As a participant in the Research and Engineering Apprenticeship program through the Army Educational Outreach office, I have been researching under Dr. Jay Hegde at Augusta University. Through my apprenticeship, I have gained a greater understanding of scientific research, visual neuroscience, and brain imaging as a whole. *andrewsojka2021@gmail.com 

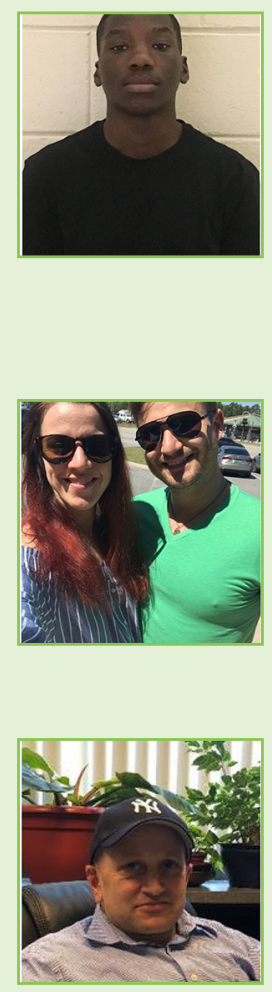

\section{SAVION HARRIS}

I am a high school junior at AR Johnson Health Science and Engineering Magnet School (Augusta, GA). While being an apprentice in the Army Educational Outreach Program (AEOP), I have been a researcher in Dr. Jay Hegdé's laboratory. Under Dr. Hegdé, I have not only learned about the brain, but also the techniques used to observe it. In my spare time, I enjoy playing soccer and long-distance running. *saviondharris@gmail.com

\section{MARISA BUCKNAM}

I am a Computer Sciences student at Augusta University in Augusta, Georgia. I am interested in studying computer programming and how we can use it to design prosthetics for the human body. When I am not studying, I am busy being a dog mom to my two wonderful pups, participating in roller derby, or finding a cool new restaurant to check out around town.

\section{JAY HEGDÉ}

Dr. Jay Hegdé is an expert in visual neuroscience at the Medical College of Georgia of Augusta University (USA). His research focuses on how the brain works under real-world conditions. He is also an Associate Editor for Frontiers in Computational Neuroscience and has recently co-edited a Research Topic issue on deep learning for multiple Frontiers journals. He spends a lot of his spare time trying to help the less fortunate, such as the fans of the Boston Red Sox. For instance, he organizes a pacifier drive for Red Sox fans on the first day of the baseball season every year, when their team is mathematically eliminated from the playoffs. 